ESAIM: PROCEEDINGS, May 2009, Vol. 27, p. 272-288

C. Besse, O. Goubet, T. Goudon \& S. Nicaise, Editors

\title{
VISCOUS PROBLEMS WITH INVISCID APPROXIMATIONS IN SUBREGIONS: A NEW APPROACH BASED ON OPERATOR FACTORIZATION
}

\author{
Martin J. Gander ${ }^{1}$, Laurence Halpern ${ }^{2}$, Caroline Japhet ${ }^{3}$ and Veronique \\ MARTIN $^{4}$
}

\begin{abstract}
In many applications the viscous terms become only important in parts of the computational domain. As a typical example serves the flow around the wing of an airplane, where close to the wing the viscous terms in the Navier Stokes equations are essential for the solution, while away from the wing, Euler's equations would suffice for the simulation. This leads to the interesting problem of finding coupling conditions between these two partial differential equations of different type. While coupling conditions have been developed in the literature, for example by using a limiting procedure on a globally viscous problem, we are interested here to develop coupling conditions which lead to coupled solutions which are as close as possible to the fully viscous solution. We develop our new approach on the one dimensional model problem of advection reaction diffusion equations with pure advection reaction approximation in subregions, which leads to the problem of coupling first and second order operators. Our guiding principle for finding transmission conditions is an operator factorization, and we show both analytically and numerically that the new coupling conditions lead to coupled solutions which are much closer to the fully viscous ones than other coupling conditions from the literature.
\end{abstract}

\section{INTRODUCTION}

There are two main reasons for coupling different models in different regions: the first are problems where the physics is different in different regions, and hence different models need to be used, for example in fluid-structure coupling. Such problems can well be treated using the so called heterogeneous domain decomposition methods, which were first presented in [19] and are methods specialized to couple different models, see also [20], [9], and in particular for fluid structure interaction [6]. The second are problems where one is in principle interested in the full physical model, but the full model is too expensive computationally over the entire region, and hence one would like to use a simpler model in most of the region, and the full one only where it is essential to capture the physical phenomena, see $[16,17]$. We are interested in the latter case here, and we use as our model problem

\footnotetext{
1 Section de Mathématiques, Université de Genève, 2-4 rue du Lièvre, CP 64, CH-1211 Genève, SWITZERLAND. martin.gander@unige.ch

${ }^{2}$ LAGA,Institut Galilée, Université Paris XIII, Rue J.B. Clément, 93430 Villetaneuse, FRANCE. halpern@math.univ-paris13.fr 3 LAGA,Institut Galilée, Université Paris XIII, Rue J.B. Clément, 93430 Villetaneuse, FRANCE. japhet@math.univ-paris13.fr ${ }^{4}$ LAMFA UMR-CNRS 6140, Université de Picardie Jules Verne, 33 Rue St. Leu, 80039 Amiens, FRANCE. veronique.martin@upicardie.fr

(c) EDP Sciences, SMAI 2009
} 
the advection reaction diffusion equation

$$
\begin{aligned}
\mathcal{L}_{a d} u:=-\nu u^{\prime \prime}+a u^{\prime}+c u & =f & & \text { in } \Omega=\left(-L_{1}, L_{2}\right), \\
\mathcal{B}_{1} u & =g_{1} & & \text { on } x=-L_{1}, \\
\mathcal{B}_{2} u & =g_{2} & & \text { on } x=L_{2},
\end{aligned}
$$

where $\nu$ and $c$ are positive constants, $a, g_{1}, g_{2} \in \mathbb{R}, f \in H^{1}(\Omega), L_{1}, L_{2}>0$ and $\mathcal{B}_{j}, j=1,2$ are suitable boundary operators of Dirichlet, Neumann or Robin type. If in part of $\Omega$, the diffusion plays only a minor role, one would like to replace the viscous solution $u$ by an inviscid approximation, which leads to two decoupled problems: a viscous problem on, say, $\Omega^{-}:=\left(-L_{1}, 0\right)$,

$$
\begin{aligned}
\mathcal{L}_{a d} u_{a d} & =f & & \text { in } \Omega^{-}, \\
\mathcal{B}_{1} u_{a d} & =g_{1} & & \text { on } x=-L_{1},
\end{aligned}
$$

and a pure advection reaction problem on $\Omega^{+}:=\left(0, L_{2}\right)$,

$$
\mathcal{L}_{a} u_{a}:=a u_{a}^{\prime}+c u_{a}=f \quad \text { in } \Omega^{+} .
$$

For this problem, coupling conditions were developed in the seminal papers [14] and for two dimensional problems in [15], and in the $\mathrm{PhD}$ thesis [7]. In [14], we find as the main motivation for the work the convenience for treating each region with an appropriate model, which can lead to substantial numerical savings. The authors develop coupling conditions between the two regions and models based on a limiting procedure for a fully viscous problem, where one would impose continuity of the solution and the fluxes, i.e.

$$
u\left(0^{-}\right)=u\left(0^{+}\right), \quad\left(-\nu^{-} u^{\prime}+a u\right)\left(0^{-}\right)=\left(-\nu^{+} u^{\prime}+a u\right)\left(0^{+}\right),
$$

where $\nu^{-}$and $\nu^{+}$are the viscosity in $\Omega^{-}$and $\Omega^{+}$. This is equivalent to imposing continuity of the solution, and continuity of the normal derivative, if $\nu$ is continuous at $x=0$.

In the case of positive advection, $a>0$, the authors in [14] find the two variational coupling conditions

$$
u_{a d}(0)=u_{a}(0), \quad\left(-\nu u_{a d}^{\prime}+a u_{a d}\right)(0)=a u_{a}(0), \quad a>0
$$

which are obtained by passing to the limit as $\nu^{+}$goes to zero in a variational formulation with the viscous coupling conditions (1.4). If the advection is negative, $a<0$, a similar analysis in [14] leads to the one variational coupling condition

$$
\left(-\nu u_{a d}^{\prime}+a u_{a d}\right)(0)=a u_{a}(0), \quad a<0,
$$

and continuity of the coupled solution is lost. The authors also introduce a different set of coupling conditions: since (1.4) implies continuity of the normal derivatives, they propose to impose directly this continuity, and not the continuity of the fluxes. This approach does not lead to a global variational formulation ("Now, the two problems do not admit a 'natural' global variational formulation and the question of existence and the asymptotic behavior are somewhat more complicated" [14]), but the authors manage to show that with these conditions, in the limit as $\nu^{+}$goes to zero, one finds for positive advection the coupling conditions

$$
u_{a d}(0)=u_{a}(0), \quad u_{a d}^{\prime}(0)=u_{a}^{\prime}(0), \quad a>0,
$$

and for negative advection

$$
u_{a d}(0)=u_{a}(0), \quad a<0,
$$

and thus the solution is at least continuous at the interface $x=0$. In a later publication [15], the authors generalize only the analysis of the variational transmission conditions to two dimensions ("Among all allowed choices, we make the most natural one, namely we take those interface conditions which are generated by a limit procedure on 'globally viscous' problems" [15]), see also [20]. 
Computational savings are also the driving force in [5], which indicates a different requirement one could try to impose on coupling conditions, namely that the coupled problem should in some sense lead to the best approximation of the fully viscous solution, an approach quite different from heterogeneous domain decomposition, since the underlying domain is homogeneous. It is known that the variational conditions do not lead to such a best approximation, and to improve the approximation, a correction boundary layer has been introduced in [4], based on singular perturbation theory, which gives in the form of matched asymptotic expansions an elegant analytical tool to treat singularly perturbed problems, see for example [21]. This analytical tool itself can be numerically exploited, in order to obtain suitable domain decomposition methods for solving problems with boundary layers, see for example [13], who name such techniques again heterogeneous domain decomposition methods, even though the same physical problem is solved throughout the domain. A different approach of viscous/inviscid coupling consists of introducing a non-linear function into the viscous term, which makes this term zero, as soon as it becomes small enough, see [3], and [1] for a numerical procedure to solve such problems in the case of Burgers equation. For more classical domain decomposition approaches for singularly perturbed problems, see [2] and references therein.

The goal of finding coupling conditions which lead to the best possible approximation of the fully viscous problem has been the focus of the $\mathrm{PhD}$ thesis [7]:

L'objectif est alors d'essayer de trouver des conditions de transmission adéquates à la frontière de façon à minimiser l'erreur entre la solution du problème de transmission et celle de Navier Stokes complet dans tout le domaine.

In one chapter of the thesis, the case of advection diffusion without reaction, $c=0$ is considered, and a set of coupling conditions is proposed: for positive advection, the conditions are

$$
u_{a d}(0)=u_{a}(0), \quad u_{a d}^{\prime}(0)=u_{a}^{\prime}(0), \quad a>0,
$$

where the second condition is obtained using a factorization of the operator in order to transfer information from the right hand side function $f$, and an expansion for $\nu$ small in order to find the original advection operator. The first condition is not mathematically justified ("puisque nous avons déjà le raccord $C^{0}$ des dérivées des solutions en $x=0$ et sachant de plus que la solution appartient à $C^{1}$, il est raisonnable de se donner cette condition" [7]). In the case of negative advection, the coupling condition is

$$
\left(-\nu u_{a d}^{\prime}+a u_{a d}\right)(0)=a u_{a}(0), \quad a<0,
$$

again obtained based on the factorization of the operator, and using this tool, it is shown that for the case $c=0$, this condition actually leads to the exact solution in the viscous part of the domain, $u_{a d} \equiv u$, provided the domain is $\Omega=\mathbb{R}$, and hence condition (1.6) can be considered as an exact transparent boundary condition for the coupled problem. We note that these latter conditions are for $a<0$ the variational conditions (V-) obtained by the limiting viscosity approach, whereas for $a>0$ these are the non-variational conditions (NV+). It has been shown in [12] for a one dimensional test problem that the non-variational coupling conditions lead to closer solutions to the fully viscous solution than the variational coupling conditions, when $\nu$ becomes small.

We will show in this paper for the model problem of an advection reaction diffusion equation in one dimension that using the factorization of the operator as a guiding principle, one can systematically construct transmission conditions which lead to solutions of the coupled problem which are very close approximations of the global viscous solution as $\nu$ goes to zero. Operator factorizations have already been successfully used in the design of other numerical methods, see for example the paraxial approximation in [18], the AILU preconditioner in [10], and in domain decomposition, see [11] and references therein. 


\section{Coupling Conditions Based on the Factorization of the Operator}

A direct computation shows that the advection reaction diffusion operator $\mathcal{L}_{a d}$ in (1.1) can be factored,

$$
\mathcal{L}_{a d}=\left(a \partial_{x}-a \lambda^{+}\right)\left(-\frac{\nu}{a} \partial_{x}+\frac{\nu}{a} \lambda^{-}\right)
$$

where $\lambda^{ \pm}=\left(a \pm \sqrt{a^{2}+4 \nu c}\right) / 2 \nu, \lambda^{+}>0$ and $\lambda^{-}<0$. In order to see how this factorization helps in finding good transmission conditions, we place ourselves first on $\Omega=\mathbb{R}$. Integrating (1.1) once for the first factor from $x$ to $\infty$, we obtain

$$
-\frac{\nu}{a} u^{\prime}(x)+\frac{\nu}{a} \lambda^{-} u(x)=-\frac{1}{a} \int_{x}^{\infty} f(\sigma) e^{\lambda^{+}(x-\sigma)} d \sigma,
$$

and thus all the information the viscous problem in $\Omega^{-}$needs from $\Omega^{+}$is the integral term over $f$ with the exponential weighting $\lambda^{+}$. Defining the modified advection reaction problem

$$
\widetilde{\mathcal{L}_{a}} \tilde{u}_{a}:=a \tilde{u}_{a}^{\prime}-a \lambda^{+} \tilde{u}_{a}=f \quad \text { in } \Omega^{+}
$$

and integrating from $x$ to $\infty$, we find that

$$
\tilde{u}_{a}(x)=-\frac{1}{a} \int_{x}^{\infty} f(\sigma) e^{\lambda^{+}(x-\sigma)} d \sigma,
$$

and thus this inviscid advection reaction problem can provide exactly the required information needed for the viscous computation on $\Omega^{-}$, it suffices to impose the coupling condition

$$
-\nu u_{a d}^{\prime}(0)+\nu \lambda^{-} u_{a d}(0)=a \tilde{u}_{a}(0)
$$

Hence after solving the modified advection reaction equation $(2.3)$ for $\tilde{u}_{a}$ on $\Omega^{+}$, we can solve the advection reaction diffusion problem on $\Omega^{-}$with coupling condition $(\mathrm{F})$ and obtain $u_{a d} \equiv u$ on $\Omega^{-}$, independent of the advection direction $a$ : this coupling condition together with the modified advection reaction equation gives the exact viscous solution on the decoupled domain $\Omega^{-}$.

If the domain is bounded, $\Omega=\left(-L_{1}, L_{2}\right)$, in addition to the information on $f$, the viscous solution on $\Omega^{-}$ also needs information from the boundary condition imposed at $x=L_{2}$. Integrating the viscous problem (1.1) from $x$ to $L_{2}$, we find that $p:=-\nu\left(u^{\prime}-\lambda^{-} u\right) / a$ is given by

$$
p(x)=p\left(L_{2}\right) e^{\lambda^{+}\left(x-L_{2}\right)}-\frac{1}{a} \int_{x}^{L_{2}} f(\sigma) e^{\lambda^{+}(x-\sigma)} d \sigma,
$$

and integrating the modified advection reaction equation (2.3) on the same interval leads to

$$
\tilde{u}_{a}(x)=\tilde{u}_{a}\left(L_{2}\right) e^{\lambda^{+}\left(x-L_{2}\right)}-\frac{1}{a} \int_{x}^{L_{2}} f(\sigma) e^{\lambda^{+}(x-\sigma)} d \sigma .
$$

As a consequence at $x=0$ the viscous solution $u$ and the inviscid solution $\tilde{u}_{a}$ are linked by the relation

$$
-\nu u^{\prime}(0)+\nu \lambda^{-} u(0)=\left(-\nu u^{\prime}\left(L_{2}\right)+\nu \lambda^{-} u\left(L_{2}\right)-a \tilde{u}_{a}\left(L_{2}\right)\right) e^{-\lambda^{+} L_{2}}+a \tilde{u}_{a}(0)
$$

Hence, if we can choose the boundary condition $\tilde{u}_{a}\left(L_{2}\right)$ for the advection reaction equation on $\Omega^{+}$such that the term $\left(u^{\prime}\left(L_{2}\right)-\lambda^{-} u\left(L_{2}\right)+a \tilde{u}_{a}\left(L_{2}\right) / \nu\right) e^{-\lambda^{+} L_{2}}$ vanishes, the coupling condition $(\mathrm{F})$ becomes again exact, and we have $u_{a d} \equiv u$ on $\Omega^{-}$. This is for example the case, if at $x=L_{2}$ the Robin condition $-\nu u^{\prime}\left(L_{2}\right)+\nu \lambda^{-} u\left(L_{2}\right)=g_{2}$ is imposed, and we choose $\tilde{u}_{a}\left(L_{2}\right)=\frac{1}{a} g_{2}$. The Robin condition $-\nu u^{\prime}\left(L_{2}\right)+\nu \lambda^{-} u\left(L_{2}\right)=g_{2}$ is in fact the 
transparent condition and leads us back to the case of the infinite line. If the advection is positive, $a>0$, then we have $\lambda^{+}=\frac{a}{\nu}+\mathcal{O}(1)$, see Lemma 3.2, and the term $e^{-\lambda^{+} L}$ is exponentially small in $\nu$ so that the choice of the boundary condition $\tilde{u}_{a}\left(L_{2}\right)$ for the advection reaction problem is not important. In the case of negative advection, $a<0$, we can use an expansion of $-\nu u^{\prime}\left(L_{2}\right)+\nu \lambda^{-} u\left(L_{2}\right)$ for $\nu$ small, see Lemma 3.6, in order to determine a suitable boundary condition $\tilde{u}_{a}\left(L_{2}\right)$, which leads in the case of a Dirichlet condition at $x=L_{2}$, $u\left(L_{2}\right)=g_{2}$, to the approximation

$$
\tilde{u}_{a}\left(L_{2}\right)=\frac{1}{a^{2}}\left(\left(c+a \lambda^{-}\right) g_{2}-f\left(L_{2}\right)\right) \nu+\frac{1}{a^{4}}\left(c^{2} g_{2}-c f\left(L_{2}\right)+a f^{\prime}\left(L_{2}\right)\right) \nu^{2}+\mathcal{O}\left(\nu^{3}\right),
$$

and in the case of a Robin condition, $\left(u^{\prime}+\alpha u\right)\left(L_{2}\right)=g_{2}$, see Lemma 3.7, we get

$$
\tilde{u}_{a}\left(L_{2}\right)=\left(\frac{\alpha+\lambda^{-}}{a \alpha-c}\left(g_{2}-\frac{1}{a} f\left(L_{2}\right)\right)\right) \nu-\frac{1}{a^{3}} \frac{\alpha+\lambda^{-}}{a \alpha-c}\left(\frac{a c^{2}}{a \alpha-c} g_{2}+\frac{a \alpha c}{a \alpha-c} f\left(L_{2}\right)+a f^{\prime}\left(L_{2}\right)\right) \nu^{2}+\mathcal{O}\left(\nu^{3}\right) .
$$

We thus propose the following algorithm to solve the coupled problem $(1.2,1.3)$ :

(1) Solve the modified advection reaction problem (2.3) on $\Omega^{+}$, for $a>0$ with boundary condition $\mathcal{B}_{2} \tilde{u}_{a}=g_{2}$ at $x=L_{2}$ (or any other convenient choice), and for $a<0$ with boundary condition $\tilde{u}_{a}\left(L_{2}\right)$ taken from the expansion $(2.8,2.9)$ up to $\mathcal{O}\left(\nu^{m}\right)$ for some $m \in \mathbb{N}$.

(2) Solve the advection reaction diffusion problem (1.2) with transmission condition (F).

(3) Solve the advection reaction problem (1.3) with $u_{a}=u_{a d}$ at $x=0$ if $a>0$, and $\mathcal{B}_{2} u_{a}=g_{2}$ at $x=L_{2}$ if $a<0$, where the latter correction is optional, since $\tilde{u}_{a}$ and $u_{a}$ are comparable for $\nu$ small.

Note that this algorithm is a direct solver, no iteration is needed, in contrast to the algorithms obtained from the variational and non-variational coupling conditions $(\mathrm{V}+)$ and $(\mathrm{NV}+)$, which require an iteration by subdomain whenever $a>0$.

\section{Rigorous Error Estimates}

We now provide rigorous error estimates for the approximation of $u_{\mid \Omega^{-}}$and $u_{\mid \Omega^{+}}$by $u_{a d}$ and $u_{a}$, both for our new coupling strategy, and the classical variational and non-variational approaches, and we use asymptotic analysis to compare them.

\subsection{Coupling Based on the Factorization}

\subsubsection{Positive Advection}

We start by studying the viscous solution $u$ of (1.1), where in the case of $a>0$, a boundary layer can appear at $x=L_{2}$. We consider at the inflow boundary $x=-L_{1}$ only the case of a Dirichlet condition, $\mathcal{B}_{1}=I d$, since the other cases lead to similar results.

Lemma 3.1. If $a>0$ and $u\left(-L_{1}\right)=g_{1}$, then the viscous solution $u$ of (1.1) satisfies at $x=0$ for $\nu$ small

$$
\begin{aligned}
u(0) & =e^{-\frac{c}{a} L_{1}} g_{1}+\frac{1}{a} \int_{-L_{1}}^{0} f(\sigma) e^{\frac{c}{a} \sigma} d \sigma+\mathcal{O}(\nu), \\
u^{\prime}(0) & =-\frac{c}{a} e^{-\frac{c}{a} L_{1}} g_{1}-\frac{c}{a^{2}} \int_{-L_{1}}^{0} f(\sigma) e^{\frac{c}{a} \sigma} d \sigma+\frac{1}{a} f(0)+\mathcal{O}(\nu),
\end{aligned}
$$


independently of the boundary condition $\mathcal{B}_{2}$ at $x=L_{2}$. At $x=L_{2}$, the derivative of $u$ has in the case of a Dirichlet condition, $u\left(L_{2}\right)=g_{2}$, the expansion

$$
u^{\prime}\left(L_{2}\right)=\frac{a}{\nu} g_{2}-\frac{a}{\nu}\left(g_{1} e^{-\frac{c}{a}\left(L_{2}+L_{1}\right)}+\frac{e^{-\frac{c}{a} L_{2}}}{a} \int_{-L_{1}}^{L_{2}} f(\sigma) e^{\frac{c}{a} \sigma} d \sigma\right)+\mathcal{O}(1) .
$$

In the case of a Robin condition, $u^{\prime}\left(L_{2}\right)+\alpha u\left(L_{2}\right)=g_{2}$, the expansions at $x=L_{2}$ for $\nu$ small are

$$
\begin{aligned}
u\left(L_{2}\right) & =g_{1} e^{-\frac{c}{a}\left(L_{2}+L_{1}\right)}+\frac{e^{-\frac{c}{a} L_{2}}}{a} \int_{-L_{1}}^{L_{2}} f(\sigma) e^{\frac{c}{a} \sigma} d \sigma+\mathcal{O}(\nu), \\
u^{\prime}\left(L_{2}\right) & =g_{2}-\alpha\left(g_{1} e^{-\frac{c}{a}\left(L_{2}+L_{1}\right)}+\frac{e^{-\frac{c}{a} L_{2}}}{a} \int_{-L_{1}}^{L_{2}} f(\sigma) e^{\frac{c}{a} \sigma} d \sigma\right)+\mathcal{O}(\nu) .
\end{aligned}
$$

Proof. Following [8], we perform an inner and outer expansion of the solution and then match them. Away from $x=L_{2}$, we seek a regular expansion of the solution $u$ for $\nu$ small, $u(x)=u_{0}(x)+\nu u_{1}(x)+\mathcal{O}\left(\nu^{2}\right)$. Using the boundary condition $u\left(-L_{1}\right)=g_{1}$ gives for the zeroth order term

$$
u_{0}(x)=g_{1} e^{-\frac{c}{a}\left(x+L_{1}\right)}+\frac{e^{-\frac{c}{a} x}}{a} \int_{-L_{1}}^{x} f(\sigma) e^{\frac{c}{a} \sigma} d \sigma .
$$

Evaluating (3.6) and its derivative at $x=0$ gives $(3.1,3.2)$.

Now for the inner expansion, we introduce the stretching variable $\xi=\left(L_{2}-x\right) / \nu$, and find for $v(\xi)=$ $u\left(\left(L_{2}-x\right) / \nu\right)$ and the Dirichlet condition $u\left(L_{2}\right)=g_{2}$ the equation

$$
\begin{aligned}
-\frac{1}{\nu} v^{\prime \prime}-\frac{a}{\nu} v^{\prime}+c v & =f, \\
v\left(L_{2}\right) & =g_{2} .
\end{aligned}
$$

Using now the regular expansion $v(\xi)=v_{0}(\xi)+\nu v_{1}(\xi)+\mathcal{O}\left(\nu^{2}\right)$, we obtain for the zeroth order term the solution

$$
v_{0}(\xi)=e^{-a \xi} g_{2}-\frac{K}{a}\left(1-e^{-a \xi}\right),
$$

with $K$ the constant which will be used in matching the inner and outer expansions.

It remains now to match the two approximate solutions (3.7) and (3.6), for which we use the matching condition $\lim _{\xi \rightarrow+\infty} v_{0}(\xi)=\lim _{x \rightarrow L_{2}} u_{0}(x)$, which yields

$$
-\frac{K}{a}=g_{1} e^{-\frac{c}{a}\left(L_{2}+L_{1}\right)}+\frac{e^{-\frac{c}{a} L_{2}}}{a} \int_{-L_{1}}^{L_{2}} f(\sigma) e^{\frac{c}{a} \sigma} d \sigma .
$$

Thus for $x$ close to $L_{2}$, we obtain the boundary layer expansion

$$
u(x)=e^{-\frac{a}{\nu}\left(L_{2}-x\right)} g_{2}+\left(g_{1} e^{-\frac{c}{a}\left(L_{2}+L_{1}\right)}+\frac{e^{-\frac{c}{a} L_{2}}}{a} \int_{-L_{1}}^{L_{2}} f(\sigma) e^{\frac{c}{a} \sigma} d \sigma\right)\left(1-e^{-\frac{a}{\nu}\left(L_{2}-x\right)}\right)+\mathcal{O}(\nu),
$$

which we can differentiate at $x=L_{2}$ to obtain (3.3).

Now in the case of Robin conditions, $v_{0}$ satisfies a homogeneous Neumann condition, $v_{0}^{\prime}(0)=0$, and we thus obtain for the zeroth order term $v_{0}=-\frac{\tilde{K}}{a}$. Using the matching condition, we find that $\tilde{K}=K$ from the Dirichlet case, and thus obtain (3.4). Finally, for (3.5), we use the boundary condition for the first order term $v_{1}$, which 
is $-v_{1}^{\prime}(0)+\alpha v_{0}(0)=g_{2}$, and taking a derivative of the expansion of $u(x)=v_{0}\left(\frac{L_{2}-x}{\nu}\right)+\nu v_{1}\left(\frac{L_{2}-x}{\nu}\right)+\mathcal{O}\left(\nu^{2}\right)$, we have $u^{\prime}\left(L_{2}\right)=-v_{1}^{\prime}(0)+\mathcal{O}(\nu)$, which together with $v_{0}=-\frac{\tilde{K}}{a}$ finishes the proof.

Before obtaining our main error estimate, we need the behavior of $\lambda^{ \pm}$for $\nu$ small, which can easily be obtained by expansion.

Lemma 3.2. For $a>0, \lambda^{ \pm}=\frac{a}{2 \nu} \pm \frac{\sqrt{a^{2}+4 \nu c}}{2 \nu}$ have for $\nu$ small the expansion

$$
\lambda^{+}=\frac{a}{\nu}+\frac{c}{a}-\frac{c^{2}}{a^{3}} \nu+\mathcal{O}\left(\nu^{2}\right), \quad \lambda^{-}=-\frac{c}{a}+\frac{c^{2}}{a^{3}} \nu+\mathcal{O}\left(\nu^{2}\right) .
$$

If $a<0$, the expansions for $\nu$ small are

$$
\lambda^{+}=-\frac{c}{a}+\frac{c^{2}}{a^{3}} \nu+\mathcal{O}\left(\nu^{2}\right), \quad \lambda^{-}=\frac{a}{\nu}+\frac{c}{a}-\frac{c^{2}}{a^{3}} \nu+\mathcal{O}\left(\nu^{2}\right) .
$$

For all $a \in \mathbb{R}$, we have

$$
\delta:=\nu\left(\lambda^{+}-\lambda^{-}\right)=|a|+\mathcal{O}(\nu) .
$$

We are now ready to prove our main error estimate in the viscous region for the case of positive advection, $a>0$. We show the result for $\mathcal{B}_{1}=I d$, similar results can also be obtained for other boundary conditions at $x=-L_{1}$.

Theorem 3.1. For $a>0, \mathcal{B}_{1}=I d$ and $\mathcal{B}_{2}$ either a Dirichlet or a Robin condition, the viscous approximation $u_{\text {ad }}$ in $\Omega^{-}$obtained with the new coupling algorithm (2.10) satisfies the estimate

$$
\left\|u-u_{a d}\right\|_{\Omega^{-}}^{2}=\nu e^{-\frac{2 a}{\nu} L_{2}} e^{-\frac{2 c}{a} L_{2}}(C+\mathcal{O}(\nu))
$$

where $C$ is a constant independent of $\nu$, and $u$ is the viscous solution of (1.1).

Proof. The error $e_{a d}:=u-u_{a d}$ satisfies in $\Omega^{-}$the equation

$$
\begin{aligned}
\mathcal{L}_{a d} e_{a d} & =0 \quad \text { in } \Omega^{-}, \\
e_{a d}\left(-L_{1}\right) & =0, \\
\left(-\nu e_{a d}^{\prime}+\nu \lambda^{-} e_{a d}\right)(0) & =\bar{K},
\end{aligned}
$$

where $\bar{K}=-\nu u^{\prime}(0)+\nu \lambda^{-} u(0)-a \tilde{u}_{a}(0)$. This equation can readily be solved, and we obtain

$$
e_{a d}(x)=-\frac{\bar{K}}{\delta}\left(e^{\lambda^{+} x}-e^{-\frac{\delta}{\nu} L_{1}} e^{\lambda^{-} x}\right) .
$$

Squaring (3.14) and integrating on $\left(-L_{1}, 0\right)$ yields

$$
\left\|e_{a d}\right\|_{\Omega^{-}}^{2}=\frac{\bar{K}^{2}}{\delta^{2}}\left(-\frac{\nu \lambda^{-}}{2 c}-\frac{2 \nu}{a} e^{-\frac{\delta}{\nu} L_{1}}-\frac{\nu \lambda^{+}}{2 c} e^{-2 \frac{\delta}{\nu} L_{1}}+\left(\frac{a}{2 c}+\frac{2 \nu}{a}\right) e^{-2 \lambda^{+} L_{1}}\right) .
$$

Using Lemma 3.2, we see that the term in the parentheses on the right is $\mathcal{O}(\nu)$, and it remains to study $\bar{K}^{2}$. Using relation (2.7) we obtain

$$
\bar{K}=\left(-\nu u^{\prime}\left(L_{2}\right)+\nu \lambda^{-} u\left(L_{2}\right)-a \tilde{u}_{a}\left(L_{2}\right)\right) e^{-\lambda^{+} L_{2}} .
$$

On the one hand, we obtain for the exponential term the expansion $e^{-\lambda^{+} L_{2}}=e^{-\frac{a}{\nu} L_{2}} e^{-\frac{c}{a} L_{2}}(1+\mathcal{O}(\nu))$. On the other hand, Lemma 3.1 shows in the case of a Dirichlet condition at $x=L_{2}$ that $-\nu u^{\prime}\left(L_{2}\right)+\nu \lambda^{-} u\left(L_{2}\right)=\mathcal{O}(1)$, and in the case of a Robin condition at $x=L_{2}$ that $-\nu u^{\prime}\left(L_{2}\right)+\nu \lambda^{-} u\left(L_{2}\right)=\mathcal{O}(\nu)$. Combining this with the estimate for the exponential, we obtain (3.13). 
In order to estimate the error in the advection part, we need several technical Lemmas, and also the solution $u_{a}$ of the advection equation (1.3),

$$
u_{a}(x)=u_{a}(0) e^{-\frac{c}{a} x}+\frac{e^{-\frac{c}{a} x}}{a} \int_{0}^{x} f(\sigma) e^{\frac{c}{a} \sigma} d \sigma .
$$

The next Lemma gives point-wise error estimates at the boundaries of $\Omega^{+}$for the case of Dirichlet conditions at $x=L_{2}$.

Lemma 3.3. If $a>0$ and $u\left(L_{2}\right)=g_{2}$, the error $e_{a}:=u-u_{a}$ of the inviscid solution $u_{a}$ obtained with the new coupling algorithm (2.10) satisfies on the boundary of $\Omega^{+}$for $\nu$ small the estimates

$$
e_{a}(0)=\mathcal{O}\left(e^{-\frac{a}{\nu}}\right), \quad e_{a}^{\prime}(0)=\mathcal{O}(\nu), \quad e_{a}\left(L_{2}\right)=\mathcal{O}(1), \quad e_{a}^{\prime}\left(L_{2}\right)=\mathcal{O}\left(\frac{1}{\nu}\right)
$$

Proof. We treat each case separately. For the first one, we have $e_{a}(0)=u(0)-u_{a}(0)=u(0)-u_{a d}(0)=e_{a d}(0)$, and thus equation (3.14) in the proof of Theorem 3.1, together with the estimates right after (3.14), give the desired result.

For the second result, using the definition of the error, we get $e_{a}^{\prime}(0)=u^{\prime}(0)-u_{a}^{\prime}(0)$, and from the advection reaction equation (1.3) we obtain $u_{a}^{\prime}(0)=\left(f(0)-c u_{a}(0)\right) / a$, with $u_{a}(0)=u_{a d}(0)=u(0)-e_{a d}(0)=u(0)+$ $\mathcal{O}\left(e^{-\frac{a}{\nu}}\right)$. Now using Lemma 3.1 for $u(0)$ and $u^{\prime}(0)$ gives the desired result.

For the third result, we get $e_{a}\left(L_{2}\right)=u\left(L_{2}\right)-u_{a}\left(L_{2}\right)=g_{2}-u_{a}\left(L_{2}\right)$, where we used the Dirichlet condition, and with (3.16) we obtain

$$
u_{a}\left(L_{2}\right)=u_{a}(0) e^{-\frac{c}{a} L_{2}}+\frac{e^{-\frac{c}{a} L_{2}}}{a} \int_{0}^{L_{2}} f(\sigma) e^{\frac{c}{a} \sigma} d \sigma
$$

which leads to the $\mathcal{O}(1)$ estimate.

For the last estimate, starting with $e_{a}^{\prime}\left(L_{2}\right)=u^{\prime}\left(L_{2}\right)-u_{a}^{\prime}\left(L_{2}\right)$, we use again Lemma 3.1 for $u^{\prime}\left(L_{2}\right)$, and from (3.16) we get

$$
u_{a}^{\prime}\left(L_{2}\right)=-\frac{c}{a} u_{a}(0) e^{-\frac{c}{a} L_{2}}-\frac{c}{a^{2}} e^{-\frac{c}{a} L_{2}} \int_{0}^{L_{2}} f(\sigma) e^{\frac{c}{a} \sigma} d \sigma+\frac{1}{a} f\left(L_{2}\right),
$$

and the term $u_{a}(0)$ can now be treated like for the second result.

Lemma 3.4. If $a>0$ and $\mathcal{B}_{2}=\partial_{x}+\alpha$, the error $e_{a}:=u-u_{a}$ of the inviscid solution $u_{a}$ obtained with the new coupling algorithm (2.10) satisfies on the boundary of $\Omega^{+}$for $\nu$ small the estimates

$$
e_{a}(0)=\mathcal{O}\left(e^{-\frac{a}{\nu}}\right), \quad e_{a}^{\prime}(0)=\mathcal{O}(\nu), \quad e_{a}\left(L_{2}\right)=\mathcal{O}(\nu), \quad e_{a}^{\prime}\left(L_{2}\right)=\mathcal{O}(1)
$$

Proof. The estimates at $x=0$ are similar to the ones proved in Lemma 3.3, since at $x=0$, the boundary operator $\mathcal{B}_{2}$ has no effect. For the estimates at $x=L_{2}$, we have $e_{a}\left(L_{2}\right)=u\left(L_{2}\right)-u_{a}\left(L_{2}\right)$, and from Lemma 3.1, we obtain $u\left(L_{2}\right)$. For $u_{a}\left(L_{2}\right)$, we can use the explicit solution formula $(3.16)$ at $x=L_{2}$, with $u_{a}(0)=u_{a d}(0)=$ $u(0)-e_{a d}(0)=u(0)+\mathcal{O}\left(e^{-\frac{a}{\nu}}\right)=g_{1} e^{-\frac{c}{a} L_{1}}+\frac{1}{a} \int_{-L_{1}}^{0} f(\sigma) e^{\frac{c}{a} \sigma} d \sigma+\mathcal{O}(\nu)$, and thus obtain that $e_{a}\left(L_{2}\right)=\mathcal{O}(\nu)$.

Finally, for $e_{a}^{\prime}\left(L_{2}\right)=u^{\prime}\left(L_{2}\right)-u_{a}^{\prime}\left(L_{2}\right)$, we obtain $u^{\prime}\left(L_{2}\right)$ from Lemma 3.1, and $u_{a}^{\prime}\left(L_{2}\right)$ from (3.18), and upon substituting $u_{a}(0)=u(0)+\mathcal{O}\left(e^{-\frac{a}{\nu}}\right)$, the result follows.

We will also need an estimate on the second derivative of the advection reaction solution, which is provided in the following Lemma.

Lemma 3.5. For $a>0$, the inviscid solution $u_{a}$ obtained with the new coupling algorithm (2.10) in $\Omega^{+}$satisfies $\left\|u_{a}^{\prime \prime}\right\|_{\Omega^{+}}^{2} \leq K_{1}$, where $K_{1}$ is independent of $\nu$. 
Proof. Squaring and integrating the equation $\mathcal{L}_{a} u_{a}^{\prime}=f^{\prime}$ on $\left(0, L_{2}\right)$ yields

$$
a^{2}\left\|u_{a}^{\prime \prime}\right\|_{\Omega^{+}}^{2}+c^{2}\left\|u_{a}^{\prime}\right\|_{\Omega^{+}}^{2}+2 a c \int_{0}^{L_{2}} u_{a}^{\prime} u_{a}^{\prime \prime}=\left\|f^{\prime}\right\|_{\Omega^{+}}^{2},
$$

and after integrating by parts, we obtain

$$
a^{2}\left\|u_{a}^{\prime \prime}\right\|_{\Omega^{+}}^{2}+c^{2}\left\|u_{a}^{\prime}\right\|_{\Omega^{+}}^{2}+a c\left(u_{a}^{\prime}\right)^{2}\left(L_{2}\right)=\left\|f^{\prime}\right\|_{\Omega^{+}}^{2}+a c\left(u_{a}^{\prime}\right)^{2}(0) .
$$

Now for the second term on the right, we can use the advection equation to obtain $u_{a}^{\prime}(0)=\left(f(0)-c u_{a}(0)\right) / a$, and by definition $u_{a}(0)=u(0)-e_{a}(0)$. By Lemma 3.1, $u(0)=\mathcal{O}(1)$, and using Lemma 3.3 and Lemma 3.4 we get $e_{a}(0)=\mathcal{O}\left(e^{-\frac{a}{\nu}}\right)$, which completes the proof.

We are now ready to state our main error estimate in the inviscid region for the case of positive advection, $a>0$. Again we show the result for $\mathcal{B}_{1}=I d$, similar results can also be obtained for other boundary conditions at $x=-L_{1}$.

Theorem 3.2. If $a>0, \mathcal{B}_{1}=I d$ and $\mathcal{B}_{2}=\partial_{x}+\alpha$, the inviscid approximation $u_{a}$ in $\Omega^{+}$obtained with the new coupling algorithm (2.10) satisfies the estimate

$$
\left\|u-u_{a}\right\|_{\Omega^{+}} \leq \mathcal{O}(\nu)
$$

where $u$ is the viscous solution of (1.1).

Proof. Since $\mathcal{L}_{a d} u_{a}=f-\nu u_{a}^{\prime \prime}$, the error $e_{a}:=u-u_{a}$ satisfies $\mathcal{L}_{a d} e_{a}=\nu u_{a}^{\prime \prime}$. Squaring this equation and integrating on $\left(0, L_{2}\right)$ yields

$$
\nu^{2}\left\|e_{a}^{\prime \prime}\right\|_{\Omega^{+}}^{2}+a^{2}\left\|e_{a}^{\prime}\right\|_{\Omega^{+}}^{2}+c^{2}\left\|e_{a}\right\|_{\Omega^{+}}^{2}-2 a \nu \int_{0}^{L_{2}} e_{a}^{\prime \prime} e_{a}^{\prime}-2 \nu c \int_{0}^{L_{2}} e_{a}^{\prime \prime} e_{a}+2 a c \int_{0}^{L_{2}} e_{a}^{\prime} e_{a}=\nu^{2}\left\|u_{a}^{\prime \prime}\right\|_{\Omega^{+}}^{2}
$$

We now integrate by parts and rearrange terms to obtain

$$
\begin{aligned}
\nu^{2}\left\|e_{a}^{\prime \prime}\right\|_{\Omega^{+}}^{2}+\left(a^{2}+2 \nu c\right)\left\|e_{a}^{\prime}\right\|_{\Omega^{+}}^{2}+c^{2}\left\|e_{a}\right\|_{\Omega^{+}}^{2}-2 a \nu \int_{0}^{L_{2}} e_{a}^{\prime \prime} e_{a}^{\prime}+a c e_{a}^{2}\left(L_{2}\right) \\
=\nu^{2}\left\|u_{a}^{\prime \prime}\right\|_{\Omega^{+}}^{2}+2 \nu c e_{a}^{\prime}\left(L_{2}\right) e_{a}\left(L_{2}\right)-2 \nu c e_{a}^{\prime}(0) e_{a}(0)+a c e_{a}^{2}(0) .
\end{aligned}
$$

Using the Cauchy Schwarz and Young's inequality, we obtain for any $\alpha>0, \beta>0$ and $\gamma>0$ the three inequalities $e_{a}^{\prime}(0) e_{a}(0) \leq \frac{1}{2 \alpha}\left(e_{a}^{\prime}\right)^{2}(0)+\frac{\alpha}{2} e_{a}^{2}(0), e_{a}^{\prime}\left(L_{2}\right) e_{a}\left(L_{2}\right) \leq \frac{1}{2 \beta}\left(e_{a}^{\prime}\right)^{2}\left(L_{2}\right)+\frac{\beta}{2} e_{a}^{2}\left(L_{2}\right)$, and $\int_{0}^{L_{2}} e_{a}^{\prime \prime} e_{a}^{\prime} \leq \frac{1}{2 \gamma}\left\|e_{a}^{\prime \prime}\right\|_{\Omega^{+}}^{2}+$ $\frac{\gamma}{2}\left\|e_{a}^{\prime}\right\|_{\Omega^{+}}^{2}$, which when inserted into (3.21) gives us the estimate

$$
\begin{aligned}
\left(\nu^{2}-\frac{a \nu}{\gamma}\right)\left\|e_{a}^{\prime \prime}\right\|_{\Omega^{+}}^{2} & +\left(a^{2}+2 \nu c-a \nu \gamma\right)\left\|e_{a}^{\prime}\right\|_{\Omega^{+}}^{2}+c^{2}\left\|e_{a}\right\|_{\Omega^{+}}^{2}+c(a-\nu \beta) e_{a}^{2}\left(L_{2}\right) \\
& \leq \nu^{2}\left\|u_{a}^{\prime \prime}\right\|_{\Omega^{+}}^{2}+\frac{\nu c}{\alpha}\left(e_{a}^{\prime}\right)^{2}(0)+\alpha \nu c e_{a}^{2}(0)+\frac{\nu c}{\beta}\left(e_{a}^{\prime}\right)^{2}\left(L_{2}\right) .
\end{aligned}
$$

Hence choosing $\gamma=\frac{a}{\nu}+\frac{c}{a}$ yields $\nu^{2}-\frac{a \nu}{\gamma}=\frac{c \nu^{3}}{a^{2}+c \nu}$ and $a^{2}+2 \nu c-a \nu \gamma=\nu c$. We also choose $\beta=\frac{a}{2 \nu}$ so that $c(a-\nu \beta)=\frac{a}{2}$ and $\frac{\nu c}{\beta}=\frac{2 c \nu^{2}}{a}$, and the constant $\alpha$ can be chosen to equal 1. We now use Lemma 3.4 and 3.5 to estimate the right hand side of (3.22), which concludes the proof. 


\subsubsection{Negative Advection}

In the case of negative advection, $a<0$, we need first a technical Lemma giving point-wise solution estimates at the boundaries of $\Omega^{+}$, for the case of Dirichlet conditions at $x=L_{2}$. We show our results for Dirichlet conditions $\mathcal{B}_{1}=I d$ at $x=-L_{1}$ only, other cases lead to similar results.

Lemma 3.6. For $a<0$ and $\mathcal{B}_{1}=\mathcal{B}_{2}=I d$, the solution $u$ of (1.1) satisfies for $\nu$ small the estimates

$$
\begin{aligned}
u(0) & =e^{\frac{c}{a} L_{2}} g_{2}-\frac{1}{a} \int_{0}^{L_{2}} f(\sigma) e^{\frac{c}{a} \sigma} d \sigma+\mathcal{O}(\nu), \\
u\left(L_{2}\right) & =g_{2}, \\
u^{\prime}(0) & =-\frac{c}{a} e^{\frac{c}{a} L_{2}} g_{2}+\frac{c}{a^{2}} \int_{0}^{L_{2}} f(\sigma) e^{\frac{c}{a} \sigma} d \sigma+\frac{1}{a} f(0)+\mathcal{O}(\nu), \\
u^{\prime}\left(L_{2}\right) & =-\frac{c}{a} g_{2}+\frac{1}{a} f\left(L_{2}\right)+\frac{\nu}{a^{3}}\left(c^{2} g_{2}-c f\left(L_{2}\right)+a f^{\prime}\left(L_{2}\right)\right)+\mathcal{O}\left(\nu^{2}\right) .
\end{aligned}
$$

Proof. Using a regular expansion of the solution $u(x)=u_{0}(x)+\nu u_{1}(x)+\mathcal{O}\left(\nu^{2}\right)$, the zeroth order term satisfies

$$
\begin{aligned}
a u_{0}^{\prime}+c u_{0} & =f \quad \text { in } \Omega, \\
u_{0}\left(L_{2}\right) & =g_{2} .
\end{aligned}
$$

The solution of this problem is $u_{0}(x)=g_{2} e^{-\frac{c}{a}\left(x-L_{2}\right)}-\frac{e^{-\frac{c}{a} x}}{a} \int_{x}^{L_{2}} f(\sigma) e^{\frac{c}{a} \sigma} d \sigma$, from which we can deduce the zeroth order terms of $u(0), u^{\prime}(0)$ and $u^{\prime}\left(L_{2}\right)$.

The first order term satisfies the equation $a u_{1}^{\prime}+c u_{1}=u_{0}^{\prime \prime}$, which implies $u_{1}^{\prime}\left(L_{2}\right)=-\frac{c}{a} u_{1}\left(L_{2}\right)+\frac{1}{a} u_{0}^{\prime \prime}\left(L_{2}\right)$, and using the boundary condition $u_{1}\left(L_{2}\right)=0$, we obtain $u_{1}^{\prime}\left(L_{2}\right)=\frac{1}{a} u_{0}^{\prime \prime}\left(L_{2}\right)=\frac{1}{a}\left(\frac{c^{2}}{a^{2}} g_{2}-\frac{c}{a^{2}} f\left(L_{2}\right)+\frac{1}{a} f^{\prime}\left(L_{2}\right)\right)$, where we differentiated the zeroth order solution twice, which concludes the proof.

Lemma 3.7. For $a<0, \mathcal{B}_{1}=I d$ and $\mathcal{B}_{2}=\partial_{x}+\alpha, \alpha \neq c / a$, the solution $u$ of (1.1) satisfies for $\nu$ small the estimates

$$
\begin{aligned}
u(0) & =\frac{a g_{2}-f\left(L_{2}\right)}{a \alpha-c} e^{\frac{c}{a} L_{2}}-\frac{1}{a} \int_{0}^{L_{2}} f(\sigma) e^{\frac{c}{a} \sigma} d \sigma+\mathcal{O}(\nu) \\
u\left(L_{2}\right) & =\frac{a g_{2}-f\left(L_{2}\right)}{a \alpha-c}-\frac{\nu}{a^{2}} \frac{1}{a \alpha-c}\left(c^{2} \frac{a g_{2}-f\left(L_{2}\right)}{a \alpha-c}-c f\left(L_{2}\right)+a f^{\prime}\left(L_{2}\right)\right)+\mathcal{O}\left(\nu^{2}\right), \\
u^{\prime}(0) & =-\frac{c}{a}\left(\frac{a g_{2}-f\left(L_{2}\right)}{a \alpha-c}\right) e^{\frac{c}{a} L_{2}}+\frac{c}{a^{2}} \int_{0}^{L_{2}} f(\sigma) e^{\frac{c}{a} \sigma} d \sigma+\frac{1}{a} f(0)+\mathcal{O}(\nu), \\
u^{\prime}\left(L_{2}\right) & =-\frac{c}{a}\left(\frac{a g_{2}-f\left(L_{2}\right)}{a \alpha-c}\right)+\frac{1}{a} f\left(L_{2}\right)+\frac{\nu}{a^{2}} \frac{\alpha}{a \alpha-c}\left(c^{2} \frac{a g_{2}-f\left(L_{2}\right)}{a \alpha-c}-c f\left(L_{2}\right)+a f^{\prime}\left(L_{2}\right)\right)+\mathcal{O}\left(\nu^{2}\right) .
\end{aligned}
$$

Proof. We proceed as in the proof of Lemma 3.6. Now $u_{0}$ satisfies the boundary condition $\left(u_{0}^{\prime}+\alpha u_{0}\right)\left(L_{2}\right)=g_{2}$ i.e. $u_{0}(x)=\frac{a g_{2}-f\left(L_{2}\right)}{a \alpha-c} e^{-\frac{c}{a}\left(x-L_{2}\right)}-\frac{e^{-\frac{c}{a} x}}{a} \int_{x}^{L_{2}} f(\sigma) e^{\frac{c}{a} \sigma} d \sigma$, which gives the zeroth order terms. The first order term $u_{1}$ satisfies now the boundary condition $\left(u_{1}^{\prime}+\alpha u_{1}\right)\left(L_{2}\right)=0$. Hence we have

$$
u_{1}(x)=-\frac{1}{a^{2}(a \alpha-c)}\left(c^{2} \frac{a g_{2}-f\left(L_{2}\right)}{a \alpha-c}-c f\left(L_{2}\right)+a f^{\prime}\left(L_{2}\right)\right) e^{-\frac{c}{a}\left(x-L_{2}\right)}-\frac{e^{-\frac{c}{a} x}}{a} \int_{x}^{L_{2}} u_{0}^{\prime \prime}(\sigma) e^{\frac{c}{a} x} d \sigma,
$$

which gives the first order terms of $u\left(L_{2}\right)$ and $u^{\prime}\left(L_{2}\right)$.

Remark 3.1. The degenerate case $\alpha=\frac{c}{a}$ can be treated as well, but we omit the details here. 
We are now ready to prove our main error estimates for the case $a<0$.

Theorem 3.3. For $a<0$ and $\mathcal{B}_{1}=\mathcal{B}_{2}=I d$, if $\tilde{u}_{a}\left(L_{2}\right)$ is chosen such that $\left(-\nu u^{\prime}\left(L_{2}\right)+\nu \lambda^{-} u\left(L_{2}\right)-a \tilde{u}_{a}\left(L_{2}\right)\right)=$ $\mathcal{O}\left(\nu^{m}\right), m \in \mathbb{N}$, see (2.8), then the viscous approximation $u_{a d}$ in $\Omega^{-}$obtained with the new coupling algorithm (2.10) satisfies the estimate

$$
\left\|u-u_{a d}\right\|_{\Omega^{-}}=\mathcal{O}\left(\nu^{m}\right)
$$

where $u$ is the viscous solution of (1.1).

Proof. Using the norm of the error $e_{a d}:=u-u_{a d}$ given in (3.15), which is also valid for $a<0$, we find with Lemma 3.2 that $-\frac{\nu \lambda^{-}}{2 c}-\frac{2 \nu}{a} e^{-\frac{\delta}{\nu} L_{1}}-\frac{\nu \lambda^{+}}{2 c} e^{-2 \frac{\delta}{\nu} L_{1}}+\left(\frac{a}{2 c}+\frac{2 \nu}{a}\right) e^{-2 \lambda^{+} L_{1}}=\mathcal{O}(1)$, and since $e^{-\lambda^{+} L_{2}}=\mathcal{O}(1)$ we obtain with the hypothesis of the theorem $\bar{K}=\left(-\nu u^{\prime}\left(L_{2}\right)+\nu \lambda^{-} u\left(L_{2}\right)-a \tilde{u}_{a}\left(L_{2}\right)\right) e^{-\lambda^{+} L_{2}}=\mathcal{O}\left(\nu^{m}\right)$, which concludes the proof.

Theorem 3.4. For $a<0$ and $\mathcal{B}_{1}=\mathcal{B}_{2}=I d$, if $\tilde{u}_{a}\left(L_{2}\right)$ is chosen such that $\left(-\nu u^{\prime}\left(L_{2}\right)+\nu \lambda^{-} u\left(L_{2}\right)-a \tilde{u}_{a}\left(L_{2}\right)\right)=$ $\mathcal{O}\left(\nu^{m}\right), m \in \mathbb{N}$, see (2.8), then the inviscid approximation $\tilde{u}_{a}$ in $\Omega^{+}$obtained with the new coupling algorithm (2.10) satisfies the estimate

$$
\left\|u-\tilde{u}_{a}\right\|_{\Omega^{+}} \leq \mathcal{O}(\nu)
$$

where $u$ is the viscous solution of (1.1).

Proof. Subtracting the two equations $\mathcal{L}_{a d} u=f$ and $\tilde{\mathcal{L}}_{a} \tilde{u}_{a}=f$ in $\Omega^{+}$, we obtain for the error $\tilde{e}_{a}:=u-\tilde{u}_{a}$ the equation

$$
a \tilde{e}_{a}^{\prime}+c \tilde{e}_{a}=\nu u^{\prime \prime}-\left(c+a \lambda^{+}\right) \tilde{u}_{a} .
$$

Multiplying this equation by $\tilde{e}_{a}$, integrating on $\Omega^{+}$and using the Cauchy-Schwarz and Young's inequality leads to

$$
c\left\|\tilde{e}_{a}\right\|_{\Omega^{+}}^{2}-\frac{a}{2} \tilde{e}_{a}^{2}(0) \leq-\frac{a}{2} \tilde{e}_{a}^{2}\left(L_{2}\right)+\frac{\nu}{2 \beta}\left\|u^{\prime \prime}\right\|_{\Omega^{+}}^{2}+\frac{\nu \beta}{2}\left\|\tilde{e}_{a}\right\|_{\Omega^{+}}^{2}+\left|c+a \lambda^{+}\right|\left(\frac{1}{2 \gamma}\left\|\tilde{u}_{a}\right\|_{\Omega^{+}}^{2}+\frac{\gamma}{2}\left\|\tilde{e}_{a}\right\|_{\Omega^{+}}^{2}\right),
$$

for any strictly positive $\beta$ and $\gamma$. If we choose $\beta=\beta_{0} / \nu$ and $\gamma=\gamma_{0} / \nu$, we get

$$
\left(c-\frac{\beta_{0}}{2}-\frac{\left|c+a \lambda^{+}\right|}{2 \nu} \gamma_{0}\right)\left\|\tilde{e}_{a}\right\|_{\Omega^{+}}^{2} \leq-\frac{a}{2} \tilde{e}_{a}^{2}\left(L_{2}\right)+\frac{\nu^{2}}{2 \beta_{0}}\left\|u^{\prime \prime}\right\|_{\Omega^{+}}^{2}+\frac{\nu\left|c+a \lambda^{+}\right|}{2 \gamma_{0}}\left\|\tilde{u}_{a}\right\|_{\Omega^{+}}^{2}
$$

Now Lemma 3.2 gives $\left|c+a \lambda^{+}\right|=\frac{c^{2}}{a^{2}} \nu+\mathcal{O}\left(\nu^{2}\right)$, so that the constants $\beta_{0}$ and $\gamma_{0}$ can be chosen such that $c-\frac{\beta_{0}}{2}-\frac{\gamma_{0}}{2}\left(\frac{c^{2}}{a^{2}}+\mathcal{O}(\nu)\right)>0$. For the first term on the right, we have $\tilde{e}_{a}\left(L_{2}\right)=u\left(L_{2}\right)-\tilde{u}_{a}\left(L_{2}\right)=g_{2}-$ $\frac{1}{a^{2}}\left(\left(c+a \lambda^{-}\right) g_{2}-f\left(L_{2}\right)\right) \nu+\mathcal{O}\left(\nu^{2}\right)=\mathcal{O}(\nu)$, and both $\left\|u^{\prime \prime}\right\|_{\Omega^{+}}^{2}$ and $\left\|\tilde{u}_{a}\right\|_{\Omega^{+}}^{2}$ can be expanded using positive powers of $\nu$, since there is no boundary layer, which leads to the announced estimate.

\subsection{Variational Coupling}

We give now rigorous error estimates for $\nu$ small for the approximations obtained by coupling the viscous and inviscid problem with the variational coupling conditions $(\mathrm{V}+)$ for $a>0$ and $(\mathrm{V}-)$ for $a<0$. Like in our previous analysis, we consider at the inflow boundary $x=-L_{1}$ only the case of a Dirichlet condition, $\mathcal{B}_{1}=I d$, since the other cases lead to similar results.

\subsubsection{Positive Advection}

Theorem 3.5. For $a>0, \mathcal{B}_{1}=I d$ and $\mathcal{B}_{2}$ either a Dirichlet or a Robin condition, the viscous approximation $u_{\text {ad }}$ in $\Omega^{-}$obtained with the variational coupling conditions $(V+)$ satisfies the estimate

$$
\left\|u-u_{a d}\right\|_{\Omega^{-}}=\mathcal{O}\left(\nu^{3 / 2}\right)
$$

where $u$ is the viscous solution of (1.1). 
Proof. The error $e_{a d}:=u-u_{a d}$ satisfies by linearity the equation

$$
\begin{aligned}
\mathcal{L}_{a d} e_{a d} & =0 \quad \text { in } \Omega^{-}, \\
e_{a d}\left(-L_{1}\right) & =0, \\
e_{a d}^{\prime}(0) & =u^{\prime}(0),
\end{aligned}
$$

where the inhomogeneous boundary condition at $x=0$ comes from the coupling conditions $(\mathrm{V}+)$, which imply $-\nu e_{a d}^{\prime}(0)+a e_{a d}(0)=-\nu u^{\prime}(0)+a u(0)-a u_{a}(0)=-\nu u^{\prime}(0)+a e_{a}(0)$, and $e_{a d}(0)=e_{a}(0)$. Solving for $e_{a d}$, we obtain

which leads to

$$
e_{a d}(x)=\frac{u^{\prime}(0)}{\lambda^{+}-\lambda^{-} e^{-\frac{\delta}{\nu} L_{1}}}\left(e^{\lambda^{+} x}-e^{-\frac{\delta}{\nu} L_{1}} e^{\lambda^{+} x}\right)
$$

$$
\left\|e_{a d}\right\|_{\Omega^{-}}^{2}=\frac{u^{\prime}(0)^{2}}{\left(\lambda^{+}-\lambda^{-} e^{-\frac{\delta}{\nu} L_{1}}\right)^{2}}\left(-\frac{\nu \lambda^{-}}{2 c}-\frac{2 \nu}{a} e^{-\frac{\delta}{\nu} L_{1}}-\frac{\nu \lambda^{+}}{2 c} e^{-2 \frac{\delta}{\nu} L_{1}}+\left(\frac{a}{2 c}+\frac{2 \nu}{a}\right) e^{-2 \lambda^{+} L_{1}}\right)=\mathcal{O}\left(\nu^{3}\right),
$$

where we used Lemma 3.1 and Lemma 3.2 for the last step.

Lemma 3.8. If $a>0, \mathcal{B}_{1}=$ Id and $\mathcal{B}_{2}=\partial_{x}+\alpha$, the error $e_{a}:=u-u_{a}$ of the inviscid approximation $u_{a}$ in $\Omega^{+}$obtained with the variational coupling conditions $(V+)$ satisfies on the boundary of $\Omega^{+}$for $\nu$ small the estimates

$$
e_{a}(0)=\mathcal{O}(\nu), \quad e_{a}^{\prime}(0)=\mathcal{O}(\nu), \quad e_{a}\left(L_{2}\right)=\mathcal{O}(\nu), \quad e_{a}^{\prime}\left(L_{2}\right)=\mathcal{O}(\nu) .
$$

Proof. We have $e_{a}(0)=u(0)-u_{a}(0)=u(0)-u_{a d}(0)=e_{a d}(0)=\frac{u^{\prime}(0)}{\lambda^{+}-\lambda^{-} e^{-\frac{\delta}{\nu} L_{1}}}\left(1-e^{-\frac{\delta}{\nu} L_{1}}\right)$, where we used (3.24) for the last step. From Lemma 3.2 and 3.3 we thus obtain the first estimate $e_{a}(0)=\mathcal{O}(\nu)$. The proof for the other three estimates is similar to the proof of Lemma 3.4, since it is based on the relations $u_{a}(0)=u_{a d}(0)=u(0)-e_{a d}(0)=u(0)+\mathcal{O}(\nu)=u_{0}(0)+\mathcal{O}(\nu)$, which still hold.

Theorem 3.6. For $a>0, \mathcal{B}_{1}=I d$ and $\mathcal{B}_{2}=\partial_{x}+\alpha$, the inviscid approximation $u_{a}$ in $\Omega^{+}$obtained with the variational coupling conditions $(V+)$ satisfies the estimate

$$
\left\|u-u_{a}\right\|_{\Omega^{+}} \leq \mathcal{O}(\nu)
$$

where $u$ is the viscous solution of (1.1).

Proof. The proof is similar to the proof of Theorem 3.2. It is based on the estimate (3.22) and uses Lemma 3.8 .

\subsubsection{Negative Advection}

Theorem 3.7. For $a<0$ and $\mathcal{B}_{1}=\mathcal{B}_{2}=I d$, the viscous approximation $u_{a d}$ in $\Omega^{-}$and the inviscid approximation $u_{a}$ in $\Omega^{+}$obtained with the variational coupling condition $\left(V_{-}\right)$satisfy the estimates

$$
\left\|u-u_{a d}\right\|_{\Omega^{-}}=\mathcal{O}(\nu), \quad\left\|u-u_{a}\right\|_{\Omega^{+}} \leq \mathcal{O}(\nu)
$$

where $u$ is the viscous solution of (1.1).

Proof. The estimate in $\Omega^{+}$is proved as in Theorem 3.4, replacing the term $c+a \lambda^{+}$by 0 . Note that in this case $-\frac{a}{2} e_{a}\left(L_{2}\right)=0$. To prove the estimate in $\Omega^{-}$, the error $e_{a d}:=u-u_{a d}$ satisfies the equation

$$
\begin{aligned}
\mathcal{L}_{a d} e_{a d} & =0, \quad \text { in } \Omega^{-}, \\
e_{a d}\left(-L_{1}\right) & =0, \\
-\nu e_{a d}^{\prime}(0)+a e_{a d}(0) & =-\nu u^{\prime}(0)+a e_{a}(0),
\end{aligned}
$$


whose solution is

$$
e_{a d}(x)=\frac{u^{\prime}(0)-\frac{a}{\nu} e_{a}(0)}{-\lambda^{-}+\lambda^{+} e^{-\frac{\delta}{\nu} L_{1}}}\left(e^{\lambda^{+} x}-e^{-\frac{\delta}{\nu} L_{1}} e^{\lambda^{-} x}\right) .
$$

Hence

$$
\left\|e_{a d}\right\|_{\Omega^{-}}^{2}=\left(\frac{u^{\prime}(0)-\frac{a}{\nu} e_{a}(0)}{-\lambda^{-}+\lambda^{+} e^{-\frac{\delta}{\nu} L_{1}}}\right)^{2}\left(-\frac{\nu \lambda^{-}}{2 c}-\frac{2 \nu}{a} e^{-\frac{\delta}{\nu} L_{1}}-\frac{\nu \lambda^{+}}{2 c} e^{-2 \frac{\delta}{\nu} L_{1}}+\left(\frac{a}{2 c}+\frac{2 \nu}{a}\right) e^{-2 \lambda^{+} L_{1}}\right) .
$$

Now the term in the parentheses on the right is $\mathcal{O}(1)$, see Lemma 3.2, and hence it remains to evaluate the term $\frac{u^{\prime}(0)-\frac{a}{\nu} e_{a}(0)}{-\lambda^{-}+\lambda^{+} e^{-\frac{\delta}{\nu} L_{1}}}$, which is $\mathcal{O}(\nu)$.

\subsection{Non-Variational Coupling}

As before, we consider only the case of a Dirichlet condition at $x=-L_{1}, \mathcal{B}_{1}=I d$, since the other cases lead to similar results.

\subsubsection{Positive Advection}

Theorem 3.8. For $a>0, \mathcal{B}_{1}=I d$ and $\mathcal{B}_{2}$ either a Dirichlet or a Robin condition, the viscous approximation $u_{a d}$ in $\Omega^{-}$obtained with the non-variational coupling conditions $(N V+)$ satisfies the estimate

$$
\left\|u-u_{a d}\right\|_{\Omega^{-}}=\mathcal{O}\left(\nu^{5 / 2}\right)
$$

where $u$ is the viscous solution of (1.1).

Proof. The boundary conditions for the error $e_{a d}:=u-u_{a d}$ in the proof of Theorem 3.5 are now $e_{a d}(0)=e_{a}(0)$ and $e_{a d}^{\prime}(0)=e_{a}^{\prime}(0)$, and we obtain

$$
e_{a d}(x)=\frac{\nu u^{\prime \prime}(0)}{a \lambda^{+}+c-\left(c+a \lambda^{-}\right) e^{-\frac{\delta}{\nu} L_{1}}}\left(e^{\lambda^{+} x}-e^{-\frac{\delta}{\nu} L_{1}} e^{\lambda^{+} x}\right) .
$$

Squaring and integrating as in Theorem 3.5 then leads to the desired estimate.

Lemma 3.9. If $\mathcal{B}_{2}=\partial_{x}+\alpha$, the error $e_{a}:=u-u_{a}$ of the inviscid approximation $u_{a}$ in $\Omega^{+}$obtained with the non-variational coupling conditions $(N V+)$ satisfies on the boundary of $\Omega^{+}$for $\nu$ small the estimates

$$
e_{a}(0)=\mathcal{O}\left(\nu^{2}\right), \quad e_{a}^{\prime}(0)=\mathcal{O}(\nu), \quad e_{a}\left(L_{2}\right)=\mathcal{O}(\nu), \quad e_{a}^{\prime}\left(L_{2}\right)=\mathcal{O}(\nu)
$$

Proof. We have $e_{a}(0)=u(0)-u_{a}(0)=u(0)-u_{a d}(0)=e_{a d}(0)=\frac{\nu u^{\prime \prime}(0)}{a \lambda^{+}+c-\left(c+a \lambda^{-}\right) e^{-\frac{\delta}{\nu} L_{1}}}\left(1-e^{-\frac{\delta}{\nu} L_{1}}\right)$, where we used (3.29) for the last step. Using Lemma 3.2 and 3.3 then yields the first estimate. The proof for the other estimates is similar to the proof of Lemma 3.4, since it is based on the relation $u_{a}(0)=u(0)+\mathcal{O}(\nu)$, which still holds.

Theorem 3.9. For $a>0, \mathcal{B}_{1}=I d$ and $\mathcal{B}_{2}=\partial_{x}+\alpha$, the inviscid approximation $u_{a}$ in $\Omega^{+}$obtained with the non-variational coupling conditions $(\mathrm{NV}+)$ satisfies the estimate

$$
\left\|u-u_{a}\right\|_{\Omega^{-}} \leq \mathcal{O}(\nu)
$$

where $u$ is the viscous solution of (1.1).

Proof. The proof is similar to the proof of Theorem 3.2, using estimate (3.22) and Lemma 3.8. 


\begin{tabular}{|c|c|c|c|}
\hline \multicolumn{4}{|c|}{$a>0$} \\
\hline & Factorization & Variational & "Non-Variational \\
\hline $\begin{array}{r}\left\|e_{a d}\right\|_{\Omega^{-}} \\
\left\|e_{a}\right\|_{\Omega^{+}} \\
\end{array}$ & $\begin{array}{c}\mathcal{O}\left(e^{-\frac{a}{\nu}}\right) \\
\mathcal{O}(\nu)\end{array}$ & $\begin{array}{c}\mathcal{O}\left(\nu^{3 / 2}\right) \\
\mathcal{O}(\nu)\end{array}$ & $\begin{array}{c}\mathcal{O}\left(\nu^{5 / 2}\right) \\
\mathcal{O}(\nu)\end{array}$ \\
\hline \multicolumn{4}{|c|}{$a<0$} \\
\hline & Factorization & Variational & "Non-Variational \\
\hline $\begin{array}{l}\left\|e_{a d}\right\|_{\Omega^{-}} \\
\left\|e_{a}\right\|_{\Omega^{+}}\end{array}$ & $\begin{array}{c}\mathcal{O}\left(\nu^{m}\right), m=1,2, \ldots \\
\mathcal{O}(\nu)\end{array}$ & $\begin{array}{l}\mathcal{O}(\nu) \\
\mathcal{O}(\nu)\end{array}$ & $\begin{array}{l}\mathcal{O}(\nu) \\
\mathcal{O}(\nu)\end{array}$ \\
\hline
\end{tabular}

TABLE 1. Summary of the asymptotic behavior of the errors for the various approaches. The integer $m$ depends on the coupling conditions chosen, see Theorem 3.3 and Theorem 3.4, and also equations (2.8) and (2.9).

\subsubsection{Negative Advection}

Theorem 3.10. For $a<0$ and $\mathcal{B}_{1}=\mathcal{B}_{2}=I d$, the viscous approximation $u_{a d}$ in $\Omega^{-}$and the inviscid approximation $u_{a}$ in $\Omega^{+}$obtained with the non-variational coupling conditions $(N V$-) satisfy the estimates

$$
\left\|u-u_{a d}\right\|_{\Omega^{-}}=\mathcal{O}(\nu), \quad\left\|u-u_{a}\right\|_{\Omega^{+}}=\mathcal{O}(\nu)
$$

where $u$ is the viscous solution of (1.1).

Proof. The solution for the variational and the non-variational coupling conditions satisfy the same equation, hence they satisfy the same estimate given by Theorem 3.7. To prove the estimate in $\Omega^{-}$, the error $e_{a d}:=u-u_{a d}$ satisfies the same equation as in the proof of Theorem 3.7 with the boundary condition $e_{a d}(0)=e_{a}(0)$, whose solution is

Hence

$$
e_{a d}(x)=\frac{e_{a}(0)}{1-e^{-\left(\lambda^{+}-\lambda^{-}\right) L_{1}}}\left(e^{\lambda^{+} x}-e^{-\frac{\delta}{\nu} L_{1}} e^{\lambda^{-} x}\right)
$$

$$
\left\|e_{a d}\right\|_{\Omega^{-}}^{2}=\left(\frac{e_{a}(0)}{1-e^{-\left(\lambda^{+}-\lambda^{-}\right) L_{1}}}\right)^{2}\left(-\frac{\nu \lambda^{-}}{2 c}-\frac{2 \nu}{a} e^{-\frac{\delta}{\nu} L_{1}}-\frac{\nu \lambda^{+}}{2 c} e^{-2 \frac{\delta}{\nu} L_{1}}+\left(\frac{a}{2 c}+\frac{2 \nu}{a}\right) e^{-2 \lambda^{+} L_{1}}\right) .
$$

Now the term in the parentheses on the right is $\mathcal{O}(1)$, see Lemma 3.2 , and hence it remains to evaluate the term $\frac{-e_{a}(0) e^{-\lambda^{-} L_{1}}}{e^{-\lambda+L_{1}-e^{-\lambda}-L_{1}}}$, which is $\mathcal{O}(\nu)$.

\subsection{Asymptotic Comparison of the Approximation Qualities}

We show in Table 1 a summary of the asymptotic results we obtained in the previous section. This comparison shows that the results from the new algorithm based on the factorization of the underlying operator are significantly better approximations of the fully viscous solution in $\Omega^{-}$than the approximations obtained with the other coupling conditions. Even in the case of negative advection, $a<0$, where the error in $\Omega^{+}$is $\mathcal{O}(\nu)$, our approximation in $\Omega^{-}$is significantly better than $\mathcal{O}(\nu)$. All these results will also be confirmed by our numerical experiments in the next section.

\section{Numerical Results}

We show now two sets of numerical experiments to illustrate our analysis. We chose $\Omega=(-1,1), \Omega^{-}=$ $(-1,0), \Omega^{+}=(0,1), f(x)=\cos x+\sin x, c=1$, and various values for the viscosity $\nu$ and the advection $a$. We discretize the advection reaction diffusion equation (1.1) using a standard second order accurate centered finite difference method, and also use a centered scheme for the pure advection reaction equation (1.3). We use 2000 

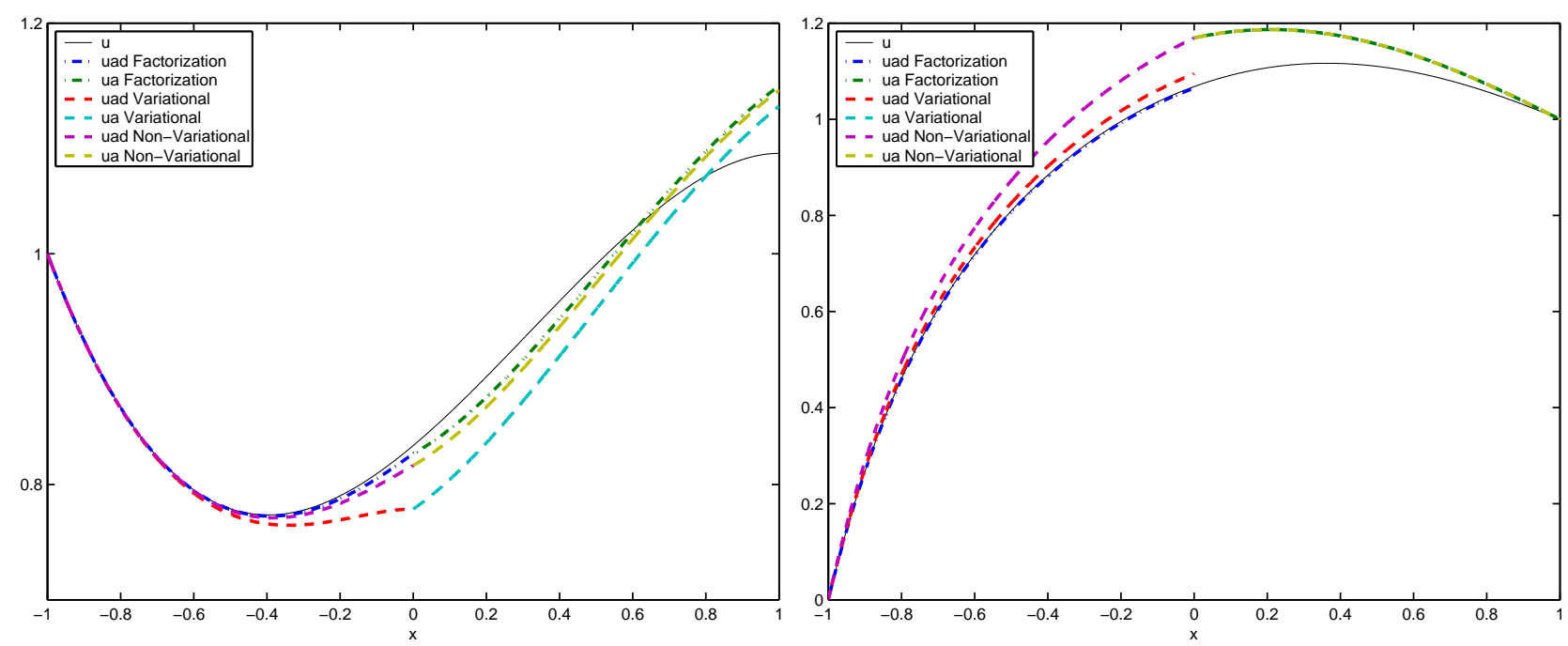

Figure 1. Approximations obtained with the various approaches, compared to the fully viscous solution, on the left for a positive advection, and on the right for a negative one.

grid points in each subregion, including boundary nodes, which leads to a discretization step $h=5.0025 e-04$ and ensures that solutions do not have spurious oscillations for the values of $\nu$ we use.

In the first set of experiments, we use a positive advection $a=1, \nu=\frac{1}{4}$, with a Dirichlet condition on the left, $u(-1)=1$, and a homogeneous Neumann condition on the right $u^{\prime}(1)=0$. In Figure 1 we show on the left the approximations obtained with the various approaches, compared to the fully viscous solution $u$. Clearly the new coupling conditions lead to the best approximation in the viscous region, followed by the nonvariational ones. The variational conditions lead to a significantly less good approximation, as we expect from our asymptotic analysis. In the inviscid region, the three approaches are comparable. Note that while the new coupling conditions lead to a non-iterative algorithm, where one simply first computes the modified advection problem, then the advection diffusion problem and finally the advection correction, both the variational and nonvariational approaches are solved by an iteration per subdomain, and we chose to relax the Dirichlet variables, in the variational case by a relaxation parameter proportional to $\nu$, and in the non-variational case by a relaxation parameter proportional to $\nu^{\frac{1}{20}}$, which turned out to lead to convergence in 7 and 8 iterations respectively.

Next we chose a negative advection, $a=-1, \nu=\frac{1}{4}$, with a homogeneous Dirichlet condition on the left, $u(-1)=0$, and an inhomogeneous Dirichlet condition on the right $u(1)=1$. In Figure 1 we show on the right the approximations obtained with the new coupling conditions, compared to the variational and non-variational approaches. Again the approximation obtained with the new coupling conditions is significantly better in the viscous region than the one obtained with the other ones, as expected from the analysis: the new approximation can hardly be distinguished in the plot from the fully viscous solution, even though in the inviscid region, the same inaccurate solution is computed. In this case, none of the algorithms requires iteration.

An asymptotic comparison of the error as a function of $\nu$ for $\nu$ small is shown in Figure 2, on the left for the case of positive advection, and on the right for negative advection. These numerical results show that the asymptotic analysis, summarized in Table 1 becomes relevant already for moderately small $\nu$, and that the new coupling conditions are largely superior for the viscous approximation qualities of the solution.

\section{Conclusions}

Using the factorization of the operator, we have been able to obtain coupling conditions for a one dimensional advection reaction diffusion equation with pure advection reaction approximation in a subregion which lead to 

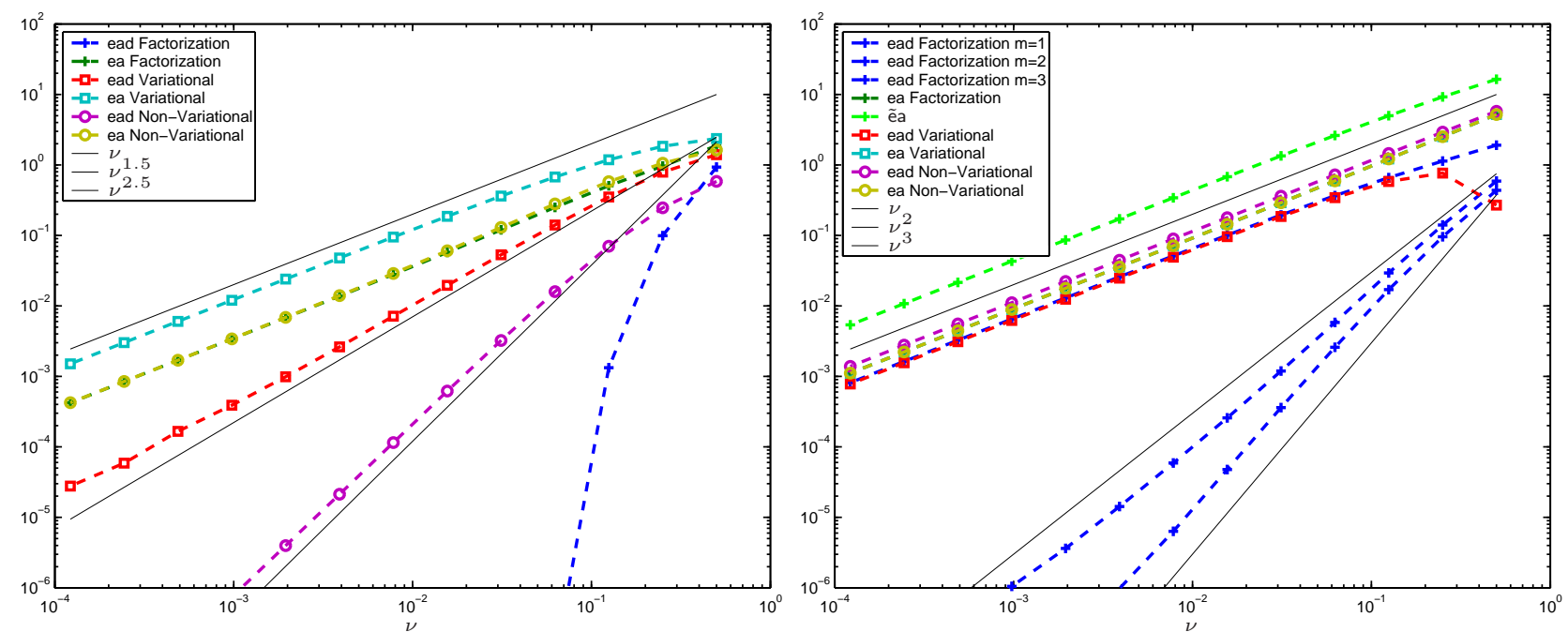

Figure 2. Asymptotic comparison of the error for the various coupling conditions, on the left for positive, and on the right for negative advection.

coupled solutions that are much closer to the fully viscous solution than any other coupling conditions from the literature. When $a>0$, the computational cost of the new algorithm is significantly lower compared to the other coupling strategies, since no iterations are needed. For $a<0$, the computational cost of the three approaches is comparable.

While the factorization approach can be generalized to higher dimensional problems and other equations, the factors will then in general contain non-local operators, which need to be approximated for convenient use as transmission conditions. In the context of coupling conditions, we will be most interested in approximations for small viscosity. As a first step into this direction, we will generalize our coupling conditions to advection reaction diffusion problems in higher dimensions.

\section{REFERENCES}

[1] R. Arina and C. Canuto. A self-adaptive domain decomposition for the viscous/inviscid coupling. I. Burgers equation. Journal of Computational Physics, 105:290-300, 1993.

[2] I. P. Boglaev. The solution of a singularly perturbed convection-diffusion problem by an iterative domain decomposition method. Numerical Algorithms, 31:27-46, 2002.

[3] F. Brezzi, C. Canuto, and A. Russo. A self-adaptive formulation for the Euler-Navier Stokes coupling. Comput. Methods Appl. Mech. Eng., 73:317-330, 1989.

[4] C. A. Coclici, G. Morosanu, and W. L. Wendland. The coupling of hyperbolic and elliptic boundary value problems with variable coefficients. Mathematical Methods in the Applied Sciences, 23:401-440, 2000.

[5] C. A. Coclici, W. L. Wendland, J. Heiermann, and M. Auweter-Kurtz. A heterogeneous domain decomposition for initialboundary value problems with conservation laws and electromagnetic fields. In T. Chan, T. Kako, H. Kawarada, and O. Pironneau, editors, Twelfth International Conference on Domain Decomposition Methods, Chiba, Japan, pages 281-288, Bergen, 2001. Domain Decomposition Press.

[6] S. Deparis, M. Discacciati, G. Fourestey, and A. Quarteroni. Heterogeneous domain decomposition methods for fluid-structure interaction problems. In O. B. Widlund and D. E. Keyes, editors, Domain Decomposition Methods in Science and Engineering XVI, volume XVI of Lecture Notes in Computational Science and Engineering 55, pages 41-52. Springer-Verlag, 2007.

[7] E. Dubach. Contribution à la Résolution des Équations fluides en domaine non borné. PhD thesis, Université Paris $13,1993$.

[8] M. V. Dyke. Perturbation Methods in Fluid Dynamics. Academic Press, New York, 1964.

[9] L. Fatone, P. Gervasio, and A. Quarteroni. Numerical solution of vascular flows by heterogeneous domain decomposition methods. In T. Chan, T. Kako, H. Kawarada, and O. Pironneau, editors, Twelfth International Conference on Domain Decomposition Methods, Chiba, Japan, pages 298-303, Bergen, 2001. Domain Decomposition Press. 
[10] M. Gander and F. Nataf. AILU: A preconditioner based on the analytic factorization of the elliptic operator. Numerical Linear Algebra with Applications, 7:543-567, 2000.

[11] M. J. Gander. Optimized Schwarz methods. SIAM J. Numer. Anal., 44(2):699-731, 2006.

[12] M. J. Gander, L. Halpern, C. Japhet, and V. Martin. Advection diffusion problems with pure advection approximation in subregions. In O. B. Widlund and D. E. Keyes, editors, Domain Decomposition Methods in Science and Engineering XVI, volume XVI of Lecture Notes in Computational Science and Engineering 55, pages 239-246. Springer-Verlag, 2007.

[13] M. Garbey and H. G. Kaper. Heterogeneous domain decomposition for singularly perturbed boundary problems. SIAM J. Numer. Anal., 34:1513-1544, 1997.

[14] F. Gastaldi and A. Quarteroni. On the coupling of hyperbolic and parabolic systems: Analytical and numerical approach. Applied Numerical Mathematics 6, 6:3-31, 1989.

[15] F. Gastaldi, A. Quarteroni, and G. S. Landriani. On the coupling of two dimensional hyperbolic and elliptic equations : Analytical and numerical approach. In T. C. et al eds., editor, Third International Symposium on Domain Decomposition Methods for Partial Differential Equations, pages 22-63, Philadelphia, 1990. SIAM.

[16] E. H. H. H. A. Schmatz. Zonal solutions for airfoils using Euler, boundary-layer and Navier-Stokes equations. In A. G. for Aerospace Research \& Development, editor, Applications of computational fluid dynamics in aeronautics: papers presented at the Fluid Dynamics Panel Symposium in Aix-en-Provence, France, number 412, pages 20-1-20-13. AGARD conference proceedings, 1986.

[17] R. Lock and B. Williams. Viscous-inviscid interactions in external aerodynamics. Prof. Aerospace Sci., 24:51-171, 1987.

[18] J. P. Lohéac, F. Nataf, and M. Schatzman. Parabolic approximations of the convection-diffusion equation. Mathematics of computation, 60:515-530, 1993.

[19] A. Quarteroni, F. Pasquarelli, and A. Valli. Heterogeneous domain decomposition principles, algorithms, applications. In D. E. Keyes, T. F. Chan, G. A. Meurant, J. S. Scroggs, and R. G. Voigt, editors, Fifth International Symposium on Domain Decomposition Methods for Partial Differential Equations, pages 129-150, Philadelphia, PA, 1992. SIAM.

[20] A. Quarteroni and L. Stolcis. Homogeneous and heterogeneous domain decomposition for compressible fluid flows at high Reynolds numbers. Numerical Methods for Fluid Dynamics, 5:113-128, 1995.

[21] E. Zauderer. Partial Differential Equations of Applied Mathematics. John Wiley \& Sons, second edition, 1989. 